\title{
Repeatability of three-dimensional thorax and pelvis kinematics in the golf swing measured using a field-based motion capture system
}

\author{
KERRIE EVANS ${ }^{1,2}$, SEAN A. HORAN ${ }^{1,2}$, ROBERT J. NEAL ${ }^{3}$, \\ ROD S. BARRETT ${ }^{1,2}$, \& PETER M. MILLS ${ }^{1,2}$
}

Q2 ${ }^{1}$ School of Physiotherapy and Exercise Science, Griffith University, Gold Coast Campus, Australia, ${ }^{2}$ Centre for Musculoskeletal Research, Griffith Health Institute, Griffith University, Gold Coast Campus, Australia, and ${ }^{3}$ Golf BioDynamics Inc., Miami, USA

(Received 14 March 2011; accepted 9 December 2011)

\begin{abstract}
Field-based methods of evaluating three-dimensional (3D) swing kinematics offer coaches and researchers the opportunity to assess golfers in context-specific environments. The purpose of this study was to establish the inter-trial, between-tester, between-location, and between-day repeatability of thorax and pelvis kinematics during the downswing using an electromagnetic motion capture system. Two experienced testers measured swing kinematics in 20 golfers (handicap $\leq 14$ strokes) on consecutive days in an indoor and outdoor location. Participants performed five swings with each of two clubs (five-iron and driver) at each test condition. Repeatability of 3D kinematic data was evaluated by computing the coefficient of multiple determination (CMD) and the systematic error (SE). With the exception of pelvis forward bend for between-day and between-tester conditions, CMDs exceeded 0.854 for all variables, indicating high levels of overall waveform repeatability across conditions. When repeatability was compared across conditions using MANOVA, the lowest CMDs and highest SEs were found for the between-tester and between-day conditions. The highest CMDs were for the inter-trial and between-location conditions. The absence of significant differences in CMDs between these two conditions supports this method of analysing pelvis and thorax kinematics in different environmental settings without unduly affecting repeatability.
\end{abstract}

Keywords: Downswing, electromagnetic system, reliability, X-Factor

\section{Introduction}

Three-dimensional (3D) motion capture systems provide a means of quantifying the kinematics of the golf swing and therefore have the potential to assist in improving understanding of factors affecting swing performance and risk for swing-related musculoskeletal injury (Hume et al., 2005). As well as having potential benefits for golfers, information acquired from $3 \mathrm{D}$ swing analysis may enhance the coaching experience and improve coaching outcomes (Wright, 2008). A variety of commercially available motion capture systems have been used to examine different aspects of the golf swing. For example, optoelectronic systems have been used to compare 
swing kinematics in different groups of golfers (Egret et al., 2006; Zheng et al., 2008a; 2008b; Tsai et al., 2010; Horan et al., 2011), assess the effect of an intervention on swing kinematics (Lephart et al., 2007), and examine factors thought to be important for golf performance and risk of injury (Myers et al., 2008; Horan et al., 2010). However, an acknowledged limitation of optoelectronic systems is that measurements are typically confined to an indoor laboratory environment, which may impose constraints on how the golfer swings the club compared to when playing or practising outdoors. In particular, the consistency of a golfer's swing in an indoor environment may be affected by environmental factors such as lighting, directional cues, and sound and perceived spatial constraints (Davids et al., 2005).

55 Q3 An alternative motion capture technology that is well suited to the outdoor environment is electromagnetic tracking, which has been used in field-based investigations of golf swing kinematics (Neal et al., 2008; Tinmark et al., 2010). The primary advantage of the electromagnetic approach for golfers and golf coaches is that it provides real-time feedback while on the course or on the practice range. The electromagnetic approach involves using electromagnetic sensors, attached to body segments of interest, to measure the 3D translation and rotation of the segments with respect to an electromagnetic transmitter. Anatomical calibration trials are performed to enable the raw sensor data to be transformed into segment anatomical coordinate systems, required for the calculation of clinically relevant segment and joint kinematics. However, a specific concern associated with the use of electromagneticbased swing analysis is that, unlike optical systems that can simultaneously capture the excursions of hundreds of markers, electromagnetic systems are constrained to a small number of sensors, typically no more than one per segment of interest. This constraint precludes the use of signal processing techniques to minimise artefact caused by the unwanted movement of the sensor with respect to the underlying skeleton (Manal et al., 2000). In addition to sensor movement, errors in the identification of anatomical landmarks (ALs) can result in errors in the calculated segment and joint kinematics (Ferber et al., 2002). Given that many experimental studies involve assessing changes in golf swing kinematics over time, and/or involve measurements made by different examiners, it is important to assess the repeatability of electromagnetic-based swing analysis. In addition, given that there are indoor and outdoor testing centres all over the world that use electromagnetic-based technology to assess golf swing kinematics, it would be of value to establish the repeatability of the approach so that the findings obtained from different locations could be compared with confidence.

Relationships between the range, sequencing, and timing of the thorax and the pelvis during the swing have been widely studied, particularly as these factors are thought to be important

80 for golf performance and risk for golf-related injury. For example, the magnitude and timing of the relative rotation between the thorax and the pelvis at the start of the downswing (termed the X-Factor) have been reported to influence short distance (McLean, 1992; Myers et al., Q4 2008) and increase the risk of low back injury (Cole \& Grimshaw, 2002; Lindsay et al., 2002). In contrast, others have reported no difference in this variable between skilled and less-skilled golfers (McTeigue et al., 1994; Egret et al., 2004; Cole \& Grimshaw, 2009) or between golfers with and without low back pain (Tsai et al., 2010). An important step in improving understanding of the significance of pelvis and thorax kinematics for the purpose of performance enhancement and injury prevention is to establish measurement reliability. We have shown that discrete thorax and pelvis kinematic measurements during the golf swing 90 acquired from an electromagnetic system in an indoor laboratory location exhibit reasonable levels of intra-tester within-session reliability with intra-class correlation coefficients ranging from 0.87 to 0.98 (Evans et al., 2008). However, to our knowledge no studies to date have assessed the repeatability of field-based 3D thorax and pelvis kinematics in indoor and outdoor locations, either between different test-sessions or between different examiners. 
The purpose of the present study was to evaluate the test-retest repeatability of 3D thorax and pelvis kinematics during the golf swing using an electromagnetic system in an indoor and outdoor location, and when measurements were made by different testers and on different days. We hypothesised that the overall test-retest repeatability of $3 \mathrm{D}$ thorax and pelvic kinematics would be high, but that the between-day and between-tester repeatability would be lower than the within-subject (inter-trial) repeatability due to additional sources of error associated with the identification of ALs.

\section{Methods}

\section{Participants}

Eighteen male and two female golfers $(M \pm S D$; age $35 \pm 9$ years, height $177 \pm 9 \mathrm{~m}$, mass $86 \pm 17 \mathrm{~kg}$, playing golf $19 \pm 10$ years, handicap $4 \pm 5$ strokes, range $-2-14$ strokes) volunteered for the study. Participants were excluded if they had sustained an injury or experienced pain in the previous 3 months that had required treatment or that prevented them from playing or practicing golf. Written informed consent was obtained prior to data collection and approval to conduct the study was given by the Institutional Human Research Ethics Committee.

\section{Testers}

Two testers, who had been trained by one of the authors ( $\mathrm{RN})$, performed all measurements. Both the testers had $>100 \mathrm{~h}$ experience in using the electromagnetic system and were familiar with the test protocol. The third person $(\mathrm{SH})$ checked if each swing trial had been captured adequately and saved the raw data so that the two testers were blinded to the trial data.

\section{Locations}

All data were collected at the Jim McLean Golf School (Miami, FL, USA). The indoor location was 5-m long, 4-m wide, and 4-m high with a 2-m square artificial turf mat placed Q3 on the floor in the centre of the room. A net at the height of the room was hung $3 \mathrm{~m}$ in front of the mat. The outdoor location was a grass teeing area on a driving range adjacent to the indoor location. The hitting area was approximately $100-\mathrm{m}$ wide and 300-m long.

\section{Protocol}

Both testers measured each participant on two occasions, one day apart. Participants performed 10 swings (five swings each with their five-iron and driver), indoors and outdoors and were measured independently by the two testers resulting in each participant performing a total of 80 swings (i.e. five swings, two clubs, two testers, two locations, and $2_{\wedge}$ days). Tester order was randomised, and location (indoor/outdoor) and club (five-iron/driver) were randomised within each tester on each day. Sensors were attached and the system was calibrated by each tester on each day. Sensors remained in place between the locations. Prior to data collection, all participants performed a standardised 10-min warm-up routine (Fradkin et al., 2004). For each swing, participants were instructed to hit the ball as straight as possible for maximum distance. Participants were instructed to pause for $30 \mathrm{~s}$ between each swing and were given $10 \mathrm{~min}$ rest between each test-retest condition. 


\section{Kinematic data collection procedure}

Kinematic data were collected at $240 \mathrm{~Hz}$ using an eight-channel Polhemus Liberty electromagnetic tracking system (Polhemus, Inc., Colchester, VT, USA) in conjunction with Golf BioDynamics software, version 1.0.1 (Golf BioDynamics Pty Ltd., Brisbane, Australia). The Polhemus Liberty system has a manufacturer-specified static accuracy of $0.762 \mathrm{~mm}$ along each sensor axis, a root-mean-square error of $0.15^{\circ}$ for sensor orientation, and a resolution of $0.038 \mathrm{~mm}$ for position and $0.0012^{\circ}$ for orientation. The electromagnetic transmitter (origin of global coordinate system) was positioned approximately $0.3 \mathrm{~m}$ behind the golfer, with $+Z$ vertically upwards, $+X$ directed away from the target and parallel to the target line, and $+Y$ directed anteriorly. Five 6-degrees-of-freedom sensors were used to record the 3D angular motion of the head, thorax, pelvis, arm, and hand. However, only data from the pelvis and thorax sensors were of interest in the present study. These sensors were placed posteriorly on the thorax at the level of T3 and the pelvis (sacrum) at the level of S2 and secured using a custom harness. All sensors were removed before being reattached and the anatomical calibration procedure was repeated for assessments made by different testers, and on different days.

Prior to collecting the kinematic data, anatomical and global (static) calibration trials were performed with the participant standing in front of the transmitter. The locations of ALs were defined within each segment's technical coordinate system defined by the sensors attached to the segments. Four ALs were used for the thorax: the lateral aspects of 4 th and 12 th ribs on the right side and the lateral aspect of the humeral heads of the left and right shoulders; and three were used for the pelvis: right and left greater trochanters and the mid-point of the left iliac crest. The position of each AL was located by sequentially placing the tip of a pointer on the landmark, and sampling position and orientation data from both the calibration sensor (pointer) and the sensor attached to the segment upon which the AL was located. In addition, a static calibration trial was collected with the subject in the anatomical position to establish the orientation of each subject's body segments in their standing posture. The origin of the thorax coordinate system was the mid-point of the two shoulder ALs, the $x$-axis was a vector directed medial-lateral from the left to right shoulder $\mathrm{AL}$, the $y$-axis was the cross product of the $x$-axis and a vector directed superiorly between the two rib ALs, and the $z$-axis was the cross product of the $x$-and $y$-axes. For the pelvis, the origin was the mid-point of the two greater trochanters, the $x$-axis was a vector directed medial-lateral from the left to right greater trochanter, the $y$-axis was the cross product of the $x$-axis and a vector directed superiorly between the left greater trochanter and left iliac crest, and the $z$-axis was the cross product of the $x$ - and $y$-axes. The GolfBioDynamics software package was then used to calculate segmental and relative joint kinematics in a manner consistent with the recommendations of the International Society of Biomechanics (Grood \& Suntay, 1983; Wu \& Cavanagh, 1995; Wu et al., 2002). For both the thorax and pelvis, angular rotations about the $x$-, $y$-, and $z$ - axes were defined as forward bend, lateral tilt, and axial rotation, respectively. In addition, thorax-pelvis separation angle or ' $\mathrm{X}$-Factor' (McLean, 1992; Hume et al., 2005) was defined as the axial rotation component of thorax motion relative to pelvis motion.

\section{Data analysis}

Data analyses were carried out using custom-designed software in Matlab version 7.8.0 (The MathWorks, Natick, MA, USA). In the present study, the top of backswing was defined as the transition point where pelvis axial rotation away from the target stopped and axial rotation towards the target began, and ball impact was defined as the time point of sudden 
deceleration of the hand sensor using a custom written algorithm. Downswing data were normalised to 101 data points for each trial using piecewise cubic spline interpolation, enabling swing data to be reported as $0-100 \%$ of the downswing cycle.

Repeatability of angular displacement-time histories was quantified using the coefficient of multiple determination (CMD) (Kadaba et al., 1989) and the systematic error (SE). The CMD is a waveform similarity statistic for which a value of 0 indicates no similarity and 1 indicates perfect agreement. CMDs were calculated for each participant from (i) the downswings of five trials for each club for day, tester, and location (intertrial); (ii) ensemble averages calculated on the same day and for the same tester from different locations (between-location); (iii) ensemble averages calculated on the same day and for the same location from different testers (between-tester); and (iv) ensemble averages calculated for the same tester and same location from different days (betweenday). The SE is a measure of the offset between time series waveforms, which uniquely contributes to the overall variability between waveforms (O'Dwyer et al., 2009). To determine the SE for angular displacements measured across different testers, locations, and days, the difference between the mean waveforms for any two respective conditions was calculated for each participant. For example, for the between-tester analysis, the mean offset between tester 1, testing indoors, on day 1 (five trials) and tester 2, testing indoors, on day 1 (five trials) was calculated first by calculating the mean waveforms for the two different conditions and then calculating the difference between the respective means of each participant. 3D pelvis and thorax angular displacement data obtained both indoors and outdoors for a representative subject using a driver are presented in Figure 1.

\section{Statistical analysis}

MANOVAs with planned contrasts were used to determine the effect of the four test-retest conditions (inter-trial, between-location, between-tester, and between-day) on the CMDs for thorax bend, thorax tilt, thorax axial rotation, pelvis bend, pelvis tilt, pelvis axial rotation, and
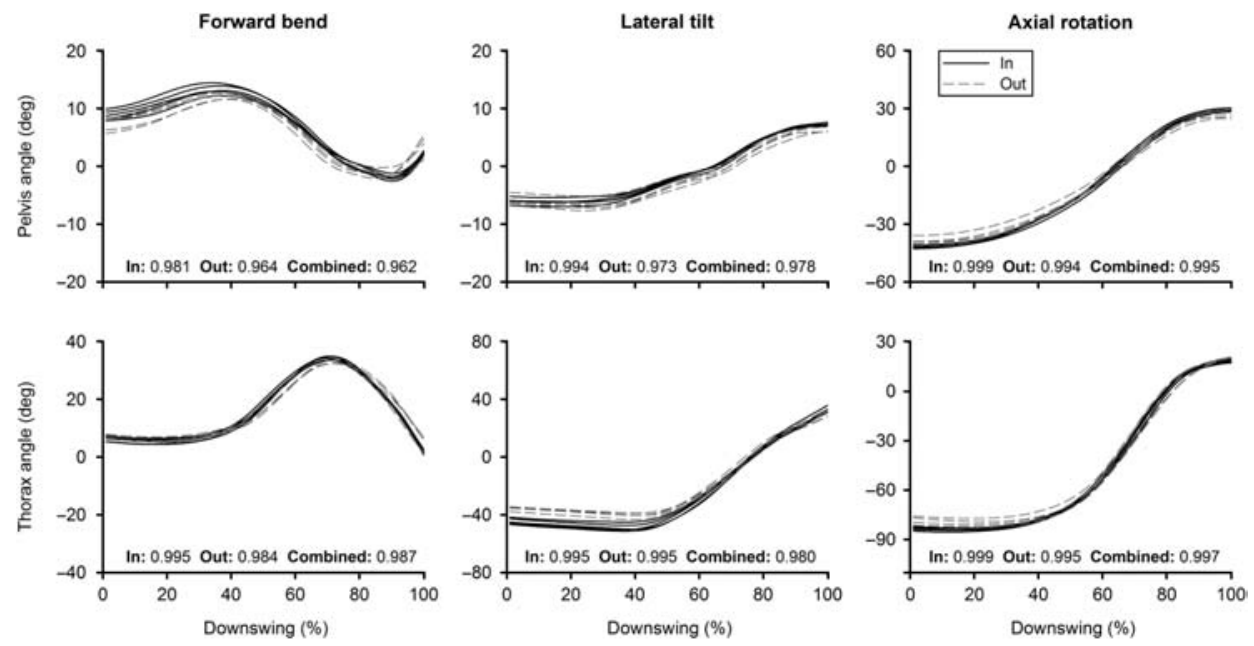

Figure 1. 3D angular displacements for the pelvis and thorax for a representative male golfer using a driver. Five indoor and five outdoor trials for pelvis and thorax forward bend, lateral tilt, and axial rotation relative to the downswing are presented. The CMD for the indoor (five trials), outdoor (five trials), and combined (inter-trial, 10 trials) conditions are displayed at the bottom of each panel. Note that different vertical axes scales have been used for the pelvis and thorax plots. 
X-Factor. All possible comparisons with respect to the inter-trial condition were made. MANOVA with planned contrasts was also used to assess the effect of three test-retest conditions (between-location, between-tester, and between-day) on the SE for the same dependent measures. All possible comparisons with respect to the inter-location condition were made. Separate MANOVAs were run for the five-iron and driver trials. Upper and lower 95\% confidence intervals (CIs) were reported for CMD data. Significance for all statistical tests was set at $p<0.05$. Analysis was carried out using SAS for Windows version 9.1.3 (SAS Institute, Inc., Cary, NC, USA).

\section{Results}

\section{Descriptive data}

CMD values ranged from 0.928 for pelvis forward bend (five-iron) to 0.997 for thorax axial rotation (five-iron and driver) for inter-trial repeatability (Table I) and from 0.817 for pelvis forward bend (five-iron) to 0.995 for thorax axial rotation (driver) for between-location repeatability (Table II). CMD values for between-testers were $\geq 0.892$ for all variables except for pelvis forward bend where the CMD value was 0.566 for swings with the five-iron and 0.628 for swings with the driver (Table III). CMD values for between-day measurements were $>0.914$ for all variables except for pelvis forward bend where the CMD value was 0.523 for swings with the five-iron and 0.602 for swings with the driver (Table IV). SE values ranged from $0.8^{\circ}$ (pelvis lateral tilt, five-iron) to $2.7^{\circ}$ (thorax axial rotation, five-iron) for between-locations (Table II), from $1.5^{\circ}$ (pelvis lateral tilt, driver) to $5.5^{\circ}$ (pelvis forward bend, five-iron; thorax axial rotation and X-Factor, five-iron; thorax axial rotation, driver) between-testers (Table III) and from $1.6^{\circ}$ (pelvis lateral tilt, driver) to $5.8^{\circ}$ (pelvis forward bend, five-iron) between-days (Table IV).

\section{MANOVA}

Test condition had a significant main effect on the CMD (five-iron: $F_{21,216}=4.12, p<0.001$; driver: $F_{21,216}=4.26, p<0.001$ ), and the SE (five-iron: $F_{14,102}=5.62, p<0.001$; driver:

Table I. Inter-trial CMD and 95\% CIs for pelvis and thorax downswing kinematics using a five-iron and driver.

Inter-trial

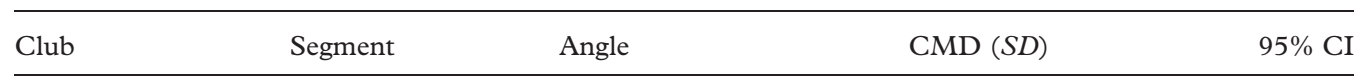

\begin{tabular}{|c|c|c|c|c|}
\hline \multirow[t]{7}{*}{ Five-iron } & \multirow[t]{3}{*}{ Pelvis } & Forward bend & $0.928(0.081)$ & $0.916-0.941$ \\
\hline & & Lateral tilt & $0.989(0.012)$ & $0.987-0.991$ \\
\hline & & Axial rotation & $0.996(0.003)$ & $0.995-0.996$ \\
\hline & \multirow[t]{4}{*}{ Thorax } & Forward bend & $0.994(0.005)$ & $0.993-0.995$ \\
\hline & & Lateral tilt & $0.996(0.003)$ & $0.996-0.997$ \\
\hline & & Axial rotation & $0.997(0.002)$ & $0.997-0.997$ \\
\hline & & X-Factor & $0.987(0.012)$ & $0.986-0.989$ \\
\hline \multirow[t]{7}{*}{ Driver } & \multirow[t]{3}{*}{ Pelvis } & Forward bend & $0.941(0.066)$ & $0.931-0.951$ \\
\hline & & Lateral tilt & $0.986(0.012)$ & $0.984-0.988$ \\
\hline & & Axial rotation & $0.996(0.003)$ & $0.996-0.997$ \\
\hline & \multirow[t]{4}{*}{ Thorax } & Forward bend & $0.992(0.008)$ & $0.990-0.993$ \\
\hline & & Lateral tilt & $0.996(0.006)$ & $0.995-0.997$ \\
\hline & & Axial rotation & $0.997(0.002)$ & $0.997-0.997$ \\
\hline & & $\mathrm{X}$-Factor & $0.985(0.014)$ & $0.983-0.987$ \\
\hline
\end{tabular}


Table II. Between-location CMD, 95\% CIs, and SE for pelvis and thorax downswing kinematics using a five-iron and driver.

Between-location

\begin{tabular}{lllllr}
\hline Club & Segment & Angle & CMD $(S D)$ & $95 \%$ CI & SE $(S D)\left({ }^{\circ}\right)$ \\
\hline Five-iron & \multirow{2}{*}{ Pelvis } & Forward bend & $0.817(0.182)$ & $0.777-0.856$ & $1.8(1.9)$ \\
& & Lateral tilt & $0.979(0.021)$ & $0.974-0.983$ & $0.8(0.9)$ \\
& & Axial rotation & $0.993(0.005)$ & $0.992-0.994$ & $2.3(1.6)$ \\
& \multirow{2}{*}{ Thorax } & Forward bend & $0.989(0.009)$ & $0.987-0.991$ & $1.3(1.4)$ \\
& & Lateral tilt & $0.991(0.008)$ & $0.989-0.993$ & $2.0(2.1)$ \\
& & Axial rotation & $0.994(0.003)$ & $0.993-0.995$ & $2.7(1.9)$ \\
Driver & X-Factor & $0.866(0.109)$ & $0.842-0.890$ & $1.8(1.9)$ \\
& \multirow{3}{*}{ Pelvis } & Forward bend & $0.854(0.167)$ & $0.817-0.890$ & $1.9(2.0)$ \\
& & Lateral tilt & $0.967(0.030)$ & $0.960-0.973$ & $1.1(0.9)$ \\
& \multirow{2}{*}{ Thorax } & Axial rotation & $0.994(0.004)$ & $0.993-0.995$ & $2.0(1.5)$ \\
& & Forward bend & $0.986(0.009)$ & $0.984-0.988$ & $1.2(0.9)$ \\
& & Lateral tilt & $0.991(0.009)$ & $0.989-0.993$ & $1.8(1.8)$ \\
& & Axial rotation & $0.995(0.004)$ & $0.994-0.996$ & $2.5(2.1)$ \\
& & X-Factor & $0.876(0.077)$ & $0.859-0.893$ & $1.8(1.8)$ \\
\hline
\end{tabular}

Table III. Between-tester CMD, 95\% CIs, and SE for pelvis and thorax downswing kinematics using a five-iron and driver.

Between-tester

\begin{tabular}{llllll}
\hline Club & Segment & Angle & CMD $(S D)$ & $95 \%$ CI & SE $(S D)\left({ }^{\circ}\right)$ \\
\hline Five-iron & \multirow{2}{*}{ Pelvis } & Forward bend & $0.566(0.302)$ & $0.500-0.632$ & $5.5(4.7)$ \\
& & Lateral tilt & $0.965(0.043)$ & $0.955-0.974$ & $1.6(1.5)$ \\
& & Axial rotation & $0.990(0.009)$ & $0.988-0.992$ & $3.0(2.2)$ \\
& \multirow{2}{*}{ Thorax } & Forward bend & $0.959(0.046)$ & $0.949-0.969$ & $3.7(3.2)$ \\
& & Lateral tilt & $0.979(0.025)$ & $0.973-0.984$ & $4.1(3.5)$ \\
& & Axial rotation & $0.985(0.011)$ & $0.983-0.988$ & $5.5(4.2)$ \\
Driver & X-Factor & $0.892(0.098)$ & $0.870-0.913$ & $5.5(4.3)$ \\
& \multirow{3}{*}{ Pelvis } & Forward bend & $0.628(0.276)$ & $0.567-0.688$ & $5.4(4.5)$ \\
& & Lateral tilt & $0.956(0.046)$ & $0.945-0.966$ & $1.5(1.4)$ \\
& \multirow{3}{*}{ Thorax } & Axial rotation & $0.992(0.006)$ & $0.991-0.993$ & $2.7(2.1)$ \\
& & Forward bend & $0.945(0.054)$ & $0.933-0.957$ & $4.1(3.4)$ \\
& & Lateral tilt & $0.976(0.029)$ & $0.970-0.983$ & $3.6(3.4)$ \\
& & Axial rotation & $0.988(0.009)$ & $0.985-0.990$ & $5.5(4.0)$ \\
& & X-Factor & $0.892(0.093)$ & $0.872-0.913$ & $5.1(4.0)$ \\
\hline
\end{tabular}

$\left.F_{14,102}=6.85, p<0.001\right)$. Planned contrasts revealed that the CMDs for inter-trial repeatability were significantly higher than for between-tester repeatability (five-iron:

$315 F_{7,70}=17.28, p<0.001$; driver: $\left.F_{7,70}=18.81, p<0.001\right)$ and between-day repeatability (five-iron: $F_{7,70}=16.13, p<0.001$; driver: $F_{7,70}=15.54, p<0.001$, Figure $2 \mathrm{~A}$ ) and that the $\mathrm{SE}$ for the between-day condition was significantly lower than that for the between-tester $\left(F_{7,51}=15.09, p<0.001\right)$ and between-location conditions $\left(F_{7,51}=12.46, p<0.001\right.$, Figure 2B). 
Table IV. Between-day CMD, 95\% CIs, and SE for pelvis and thorax downswing kinematics using a five-iron and driver.

Between-day

320

\section{Discussion}

The present study evaluated the test-retest repeatability of $3 \mathrm{D}$ thorax and pelvis kinematics during the golf downswing using a commercially available electromagnetic motion tracking system in an indoor and outdoor environment, and with different testers on consecutive days. The main finding of the study was that the overall repeatability of thorax and pelvic kinematic measures, including X-Factor, was high irrespective of whether the measurements were made indoors or outdoors, by different testers or on different days. CMDs exceeded conditions. We also found, consistent with our hypothesis, that the repeatability of kinematic measures with both five-iron and driver was lowest for between-day and between-tester conditions, which we attributed to errors associated with inconsistent re-identification of ALs under test-retest conditions. 


\section{Inter-trial repeatability}

As expected, the highest CMDs in the present study were obtained when repeatability was assessed between the trials performed by each participant. With the exception of pelvis forward bend (mean CMD =0.93), all inter-trial CMDs were above 0.98, suggesting that participants were able to closely replicate the same swing kinematics across multiple trials performed at each condition. The participants in the present study were considered to have the heterogeneity needed to examine test-retest swing repeatability (Bartlett \& Frost, 2008) and also to possess the range of characteristics that would reflect those golfers likely to undergo 3D swing analysis. That is, participants had golf handicaps ranging between -2 and 14 strokes; had been playing golf for between 7 and 40 years; were aged between 19 and 51 years; and had a body mass index of between 22 and $37 \mathrm{~kg} / \mathrm{m}^{2}$. The findings from the present study can therefore be generalised to golfers with the aforementioned characteristics. The high CMDs for inter-trial repeatability also suggest that measurement errors associated with the movement of the electromagnetic sensors relative to the underlying segments (i.e. thorax and pelvis) were at best small, or at worst resulted in a consistent bias that did not adversely affect inter-trial repeatability.

\section{Between-location repeatability}

Given the potential for differences in environmental conditions between indoor and outdoor locations (e. g. perceived spatial constraints, lighting, and surface), it was of interest in the present study to determine whether location affected the repeatability of swing kinematics relative to inter-trial repeatability. Our finding of no difference between inter-location and inter-trial CMD suggests that location does not give rise to any additional variability that is not already explained at the level of inter-trial variability. From a practical point of view, this indicates that the location (indoor versus outdoor) does not affect the ability to reliably assess golf swing kinematics, and so indoor and outdoor testings can be considered equivalent for this purpose.

\section{Between-tester and between-day repeatability}

With the exception of pelvis forward bending (CMDs $=0.52-0.63$ ), CMDs were $>0.89$ for between-tester and between-day conditions, indicating high repeatability. However, the CMDs for the between-tester and between-day conditions were significantly lower than the inter-trial condition. Similarly, the SE was significantly higher for between-tester and between-day compared to the between-location conditions. Given that between-tester and between-day comparisons required re-identification of ALs by different testers and on different days, our findings are most likely explained by the variation in landmark identification between these test-retest conditions. This finding is consistent with kinematic studies of other motor tasks such as gait, where marker reapplication and landmark identification errors are considered to be the key factors in decreasing measurement repeatability (Schwartz et al., 2004; Mills et al., 2007; Monaghan et al., 2007; McGinley et al., 2009). The implication of the findings from the present study is that when swing kinematics are compared under test-retest conditions (i.e. different examiners on the same day or same examiner on different days), at least part of the observed difference in swing kinematics may be attributable to the sources of variability unrelated to the swing.

A consistent finding from the present study was that CMDs tended to be lowest and the SE to be highest for forward bending of the pelvis. There are several possible explanations 
for this finding. First, the ALs on the pelvis and hips (i.e. greater trochanters and mid-point of iliac crest) were difficult to locate in some participants due to the amount and relative motion of overlying tissue, and errors in subjective identification of these landmarks would tend to adversely affect the CMDs and SEs for conditions in which the sensors and

410 landmarks were redefined (i.e. between-tester and between-day conditions). Second, the manner in which CMDs are calculated results in higher CMDs for waveforms with a greater range (McGinley et al., 2009), and during the downswing the range of motion of pelvis forward bend is typically small compared to the range of motion for the other degrees of freedom assessed. Use of alternative landmarks to define the anatomical coordinate system for the pelvis (e.g. Mills et al., 2007) may result in improved repeatability for pelvis forward bend.

\section{Conclusion}

420 The repeatability of thorax and pelvic golf swing kinematics was generally high, irrespective of whether the measurements were made indoors or outdoors, by different testers or on different days. The absence of significant differences in CMDs between the inter-trial and betweenlocation conditions supports the use of this field-based method of analysing pelvis and thorax kinematics in different environmental settings without unduly affecting repeatability. Efforts to improve repeatability of golf swing kinematics assessed on different days and by different testers should focus on reducing errors associated with the re-identification of ALs under test-retest conditions, especially for the pelvis.

\section{Acknowledgements}

This project was supported by the Griffith University Industry Collaborative Scheme. The authors wish to thank the staff at the Jim McLean Golf School in Miami, Florida, USA for allowing this research to be carried out at their facilities.

435 Conflict of interest: This study was supported by the Griffith University Industry Collaborative Scheme with Golf BioDynamics Inc. Dr Neal is a Director of Golf BioDynamics Inc.

\section{References}

Bartlett, J. W., \& Frost, C. (2008). Reliability, repeatability and reproducibility: Analysis of measurement errors in continuous variables. Ultrasound in Obstetrics and Gynaecology, 31, 466-475.

Chu, Y., Sell, T. C., \& Lephart, S. M. (2010). The relationship between biomechanical variables and driving performance during the golf swing. Fournal of Sports Sciences, 28, 1251-1259.

Cole, M. H., \& Grimshaw, P. N. (2009). The X-Factor and its relationship to golfing performance. Fournal of Quantitative Analysis in Sports, 5, 1-19.

Davids, K., Bennett, S. J., \& Newell, K. M. (Eds.) (2005). Movement system variability. Champaign, IL: Human Kinetics.

Egret, C. I., Dujardin, F. H., Weber, J., \& Chollet, D. (2004). 3-D kinematic analysis of the golf swings of expert and experienced golfers. Fournal of Human Movement Studies, 47, 193-204. Retrieved from http://www.getcited.org/ $\mathrm{pub} / 100488246$

Egret, C. I., Nicolle, B., Dujardin, F. H., Weber, J., \& Chollet, D. (2006). Kinematic analysis of the golf swing in men and women experienced golfers. International fournal of Sports Medicine, 27, 463-467.

Evans, K., Refshauge, K. M., Adams, R. D., \& Barrett, R. (2008). Swing kinematics in skilled male golfers following putting practice. Fournal of Orthopaedic and Sports Physical Therapy, 38, 425-433.

Ferber, R., McClay Davis, I., Williams, D. S., \& Laughton, C. (2002). A comparison of within- and between-day reliability of discrete 3D lower extremity variables in runners. Fournal of Orthopaedic Research, 20, 1139-1145. 
Fradkin, A. J., Sherman, C. A., \& Finch, C. F. (2004). Improving golf performance with a warm up conditioning programme. British fournal of Sports Medicine, 38, 762-765.

Grood, E. S., \& Suntay, W. J. (1983). A joint coordinate system for the clinical description of three-dimensional motions: Application to the knee. Fournal of Biomechanical Engineering, 105, 136-144.

Horan, S. A., Evans, K., Morris, N. R., \& Kavanagh, J. J. (2010). Thorax and pelvis kinematics during the downswing of male and female skilled golfers. Fournal of Biomechanics, 43, 1456-1462.

Horan, S. A., Evans, K., \& Kavanagh, J. J. (2011). Movement variability in the golf swing of male and female skilled golfers. Medicine E Science in Sports E Exercise, 43, 1474-1483.

Hume, P. A., Keogh, J., \& Reid, D. (2005). The role of biomechanics in maximising distance and accuracy of golf shots. Sports Medicine, 35, 429-449.

Kadaba, M. P., Ramakrishnan, H. K., Wootten, M. E., Gainey, J., Gorton, G., \& Cochran, G. V. (1989). Repeatability of kinematic, kinetic, and electromyographic data in normal adult gait. Fournal of Orthopaedic Research, 7, 849-860.

Lephart, S. M., Smoliga, J. M., Myers, J. B., Sell, T. C., \& Tsai, Y. S. (2007). An eight-week golf-specific exercise program improves physical characteristics, swing mechanics, and golf performance in recreational golfers. Fournal of Strength and Conditioning Research, 21, 860-869.

Lindsay, D. M., Horton, J. F., \& Paley, R. D. (2002). Trunk motion of male professional golfers using two different golf clubs. Fournal of Applied Biomechanics, 18, 366-373. Retrieved from http://journals.humankinetics.com/jab.

Manal, K., McClay, I., Stanhope, S., Richards, J., \& Galinat, B. (2000). Comparison of surface mounted markers and attachment methods in estimating tibial rotations during walking: An in vivo study. Gait $\mathcal{G}$ Posture, 11, $38-45$.

McGinley, J. L., Baker, R., Wolfe, R., \& Morris, M. E. (2009). The reliability of three-dimensional kinematic gait measurements: A systematic review. Gait $\mathcal{E}$ Posture, 29, 360-369.

McLean, J. (1992). Widen the gap. Golf Magazine, 12, 49-53.

470 McTeigue, M., Lamb, S. R., Mottram, R., \& Pirozzolo, F. (1994). Spine and hip motion analysis during the golf swing. In A. J. Cochran, and M. R. Farrally (Eds.), Science and Golf II: Proceedings of the World Scientific Congress of Golf (pp. 50-58). London: E \& FN Spon.

Mills, P. M., Morrison, S., Lloyd, D. G., \& Barrett, R. S. (2007). Repeatability of 3D gait kinematics obtained from an electromagnetic tracking system during treadmill locomotion. Fournal of Biomechanics, 40, 1504-1511.

Monaghan, K., Delahunt, E., \& Caulfield, B. (2007). Increasing the number of gait trial recordings maximises intrarater reliability of the CODA motion analysis system. Gait $\mathcal{E}$ Posture, 25, 303-315.

Myers, J., Lephart, S., Tsai, Y. S., Sell, T., Smoliga, J., \& Jolly, J. (2008). The role of upper torso and pelvis rotation in driving performance during the golf swing. Fournal of Sports Science, 26, 181-188.

Neal, R. J., Lumsden, R. G., Holland, M., \& Mason, B. (2008). Segment interactions: Sequencing and timing in the downswing. In D. Crews, and R. Lutz (Eds.), Science and Golf V: Proceedings of the World Scientific Congress of Golf (pp. 21-29). Mesa, AZ: Energy in Motion Inc.

O’Dwyer, N., Smith, R., Halaki, M., \& Rattanaprasert, U. (2009). Independent assessment of pattern and offset variability of time series waveforms. Gait $\mathcal{E}$ Posture, 29, 285-289.

Schwartz, M. H., Trost, J. P., \& Wervey, R. A. (2004). Measurement and management of errors in quantitative gait data. Gait \& Posture, 20, 196-203.

Tinmark, F., Hellstrom, J., Halvorsen, K., \& Thorstensson, A. (2010). Elite golfers' kinematic sequence in fullswing and partial-swing shots. Sports Biomechanics, 9, 236-244.

Tsai, Y. S., Sell, T. C., Smoliga, J. M., Myers, J. B., Learman, K. E., \& Lephart, S. M. (2010). A comparison of physical characteristics and swing mechanics between golfers with and without a history of low back pain. Fournal of Orthopaedic and Sports Physical Therapy, 40, 430-438.

Wright, I. (2008). Motion capture in golf. International fournal of Sports Science and Coaching, 3, $161-182$.

Wu, G., \& Cavanagh, P. R. (1995). ISB recommendations for standardization in the reporting of kinematic data. Fournal of Biomechanics, 28, 1257-1261.

490 Wu, G., Siegler, S., Allard, P., Kirtley, C., Leardini, A., Rosenbaum, D., ... Stokes, I. (2002). ISB recommendation on definitions of joint coordinate system of various joints for the reporting of human joint motion - part I: Ankle, hip, and spine. International Society of Biomechanics. Fournal of Biomechanics, 35, 543-548.

Zheng, N., Barrentine, S. W., Fleisig, G. S., \& Andrews, J. R. (2008a). Kinematic analysis of swing in pro and amateur golfers. International fournal of Sports Medicine, 29, 487-493.

Zheng, N., Barrentine, S. W., Fleisig, G. S., \& Andrews, J. R. (2008b). Swing kinematics for male and female pro golfers. International fournal of Sports Medicine, 29, 965-970. 